Annales Geophysicae (2001) 19: 655-666 (c) European Geophysical Society 2001

(5)

\title{
Disturbance of plasma environment in the vicinity of the Astrid-2 microsatellite
}

\author{
N. Ivchenko ${ }^{1}$, L. Facciolo ${ }^{1,2}$, P. A. Lindqvist ${ }^{1}$, P. Kekkonen ${ }^{3}$, and B. Holback ${ }^{4}$ \\ ${ }^{1}$ Alfvén Laboratory, Royal Institute of Technology, Stockholm, Sweden \\ ${ }^{2}$ Politecnico di Torino, C. so Duca degli Abruzzi, Torino, Italy \\ ${ }^{3}$ Oulu University, Oulu, Finland \\ ${ }^{4}$ Swedish Institute of Space Physics, Uppsala, Sweden
}

Received: 19 July 2000 - Revised: 20 November 2000 - Accepted: 15 December 2000

\begin{abstract}
The presence of a satellite disturbs the ambient plasma. The charging of the spacecraft creates a sheath around it, and the motion of the satellite creates a wake disturbance. This modification of the plasma environment introduces difficulties in measuring electric fields and plasma densities using the probe technique. We present a study of the structure of the sheath and wake around the Astrid2 microsatellite, as observed by the probes of the EMMA and LINDA instruments. Measurements with biased LINDA probes, as well as current sweeps on the EMMA probes, show a density enhancement upstream of the satellite and a plasma depletion behind the satellite. The electric field probes detect disturbances in the plasma potential on magnetic field lines connected to the satellite.
\end{abstract}

Key words. Space plasma physics (spacecraft sheaths, wakes, charging; instruments and techniques)

\section{Introduction}

Satellites are widely used for in situ measurements in the ionosphere and the magnetosphere of the Earth. To make detailed measurements it is necessary to understand the disturbances the instrument (in this case, the satellite itself) introduces in the studied system. A body in contact with a plasma usually becomes charged with respect to the plasma potential. The charge is determined by the balance of currents both to and from the body, carried by plasma electrons and ions impinging on the body, and photoelectrons and secondary electrons emitted by the body itself. To achieve a current balance, the potential distribution around the body is disturbed and thus, attracts or reflects particles of the corresponding charge. A body moving through a compressive medium disturbs the density and velocity distributions around it, forming a wake region behind it and a compression in front of it.

Among the basic measurement principles the Langmuir probe holds an important place. Used in the laboratory since

Correspondence to: N. Ivchenko (ivchenko@plasma.kth.se) the 1920's, it has also been applied successfully on satellites. The most common applications are the double probe technique for measuring electric fields (Fahleson, 1967), biased Langmuir probes for density measurements (e.g. Eriksson and Boström, 1995), and current/voltage swept probes for determining plasma parameters (Fahleson et al., 1974). Ideally, one would like to expose the probes to the undisturbed plasma, but in reality, the plasma is disturbed by the satellite to which the probes are attached by means of wire booms. The probes are kept away from the satellite by the centrifugal force due to the satellite spin.

Studies of the interaction of the spacecraft with the space plasma environment have been conducted both for practical and fundamental reasons since the beginning of the space age. A recent review by Hastings (1995) gives an idea of the relative amount of research done on various aspects of the spacecraft-plasma interaction. The most direct phenomenon to study is the formation of the wake (Al'pert et al., 1965; Kasha, 1969). Several in situ measurements have been reported (e.g. Samir and Jew, 1972), and compared with different theories (Samir and Fontheim, 1981; Samir et al., 1989). Another important subject of research is that of spacecraft charging (Garrett, 1985) which, in extreme cases, may lead to dramatic effects on the spacecraft instrumentation. The advent of the Space Shuttle opened possibilities to study the environment of larger bodies in orbit (Murphy et al., 1989). Other recent studies have addressed current collection by highly charged spacecrafts (Neubert et al., 1990), an important question for the understanding of the charging of bodies in the wake of larger spacecraft and electrodynamics of tethered systems (Dobrowolny and Melchioni, 1993). There has been surprisingly little work done on understanding the influence of the satellite-related disturbances on the measurements with Langmuir probes in space plasmas.

Among recent reports focusing on the possible effect of the satellite-related disturbances of the plasma environment, the studies based on data from Viking and Freja satellites should be mentioned. Hilgers et al. (1992) reported on Viking Langmuir probe measurements, and found a dependence of the 


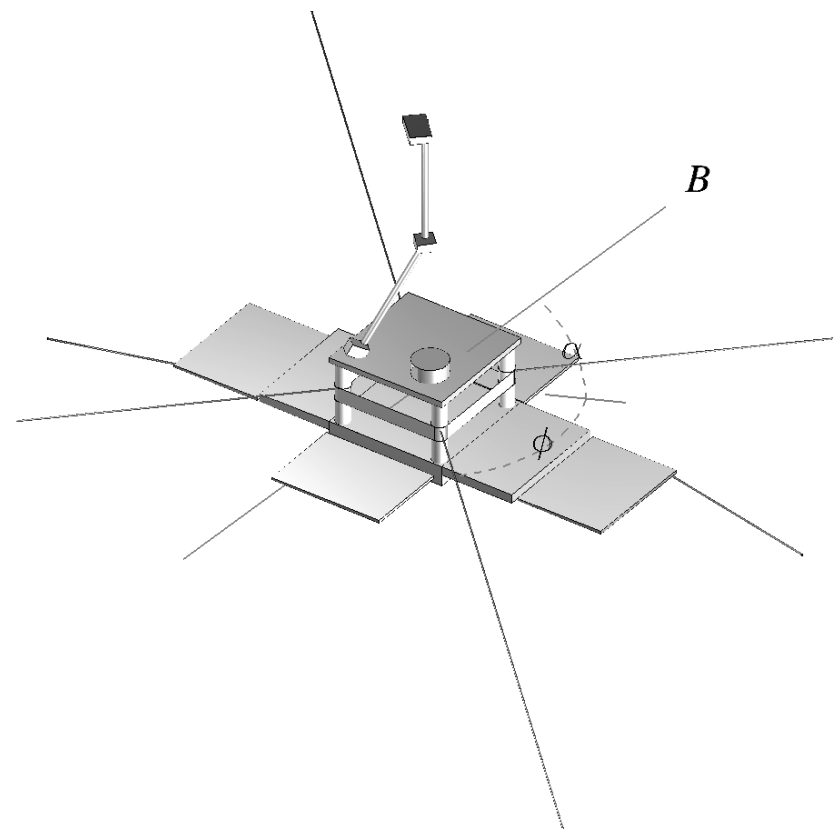

Fig. 1. Schematics of the Astrid-2 microsatellite. LINDA probes are mounted at the stiff booms protruding from the solar panel edges, with probe $n 1$ on the right side of the figure. The EMMA probes (not shown) are placed at the ends of the wire booms, and numbered clockwise, starting with probe 1 in the top of the figure. $\alpha$ is the angle between the magnetic field and the spin plane, and spin phase $\phi$ is measured between the direction of probe 3 and projection of the magnetic field in the spin plane.

probe current on the angle to the magnetic field. On Viking altitudes, the densities are low and photoelectrons play a significant role (Hilgers and Holback, 1993). A theory describing the dynamics of ambient electrons and photoelectrons was developed by Hilgers (1995). Disturbances of the electric field measurements due to the gradients of the background plasma density were discussed by Laakso et al. (1995). Chust et al. (1998) addressed the question of the anomalously strong electric field parallel to the magnetic field, which is occasionally measured on Freja, and demonstrated that it was not related to either particle shadowing or density gradients. They were not able to exclude other mechanisms that could have produced similar artefacts.

The purpose of this work is to study the plasma environment of the Astrid-2 microsatellite by means of two Langmuir probe instruments onboard - the EMMA and LINDA instruments. Astrid-2 (see Fig. 1) was launched on 11 December 1998 into a $83^{\circ}$ declination circular orbit at $1000 \mathrm{~km}$ altitude and was operational until the end of July 1999. As the LINDA instrument is described in detail elsewhere in this issue we will present here only their most important characteristics. The EMMA instrument for measuring the electric field consists of four identical spherical probes $(40 \mathrm{~mm}$ in diameter) placed $3.3 \mathrm{~m}$ away from the satellite center at the tips of wire booms. The satellite was spin-stabilized, with the spin period around 7 seconds, and spin axis oriented to- wards the Sun. The potentials of all four probes were measured relative to the satellite to derive the electric field. There was a possibility to feed a bias current to the probes. During the first two months of the mission, no bias current was fed to the EMMA probes and for the remainder of the mission, the bias current to the probes was set to $+20 \mathrm{nA}$. In a special sweep mode, the bias current could be switched quickly through a commanded set of values, and the probe potentials provided the current-voltage characterstics, from which the plasma parameters can be derived. The LINDA instrument measured the electron current collected by two smaller probes (diameter of $10 \mathrm{~mm}$ ), located on stiff booms mounted on the solar panels of the satellite $1.45 \mathrm{~m}$ away from the centre of the satellite. The LINDA probes were biased positive $\left(U_{\text {bias }}=+10 \mathrm{~V}\right)$ relative to the satellite, and thus, should collect electrons effectively, with the value of the current being proportional to the electron density. The collected current also weakly depends on the electron temperature, which is normally assumed constant when interpreting the data. The surface of one of the LINDA probes - $n 1$ - was probably contaminated during launch, and only the measurements from the other probe - $n 2$ - will be used here.

This paper proceeds as follows. First, we describe the geometry of the measurements, including the satellite configuration with respect to the orbital plane and the ambient magnetic field. Later we present several cases for which three types of measurements were conducted - the EMMA probe potentials, current sweeps on one of the EMMA probes and LINDA continuous current measurements. The results are discussed and summarized in the last section.

\section{Geometry of the measurements}

The configuration of the Astrid-2 satellite means that measurements are possible only in the spin plane, at a constant distance from the satellite determined by the boom length. The disturbances of the plasma environment can only be detected if the plasma parameters at a probe location change with the spin phase, i.e. any symmetric sheaths or those rotating together with the satellite cannot be studied in-flight.

The geometry is, however, complicated. The satellite shape is far from being spherically symmetric. There are at least three externally imposed directions which are expected to break the symmetry of the sheath: the magnetic field, the spacecraft velocity and the direction towards the Sun. The ambient magnetic field $\boldsymbol{B}$ will affect the motion of the plasma particles. The electron gyroradius is of the order of several $\mathrm{cm}$, which is much smaller than the satellite size. The ion gyroradii are much larger for all the ion species, and ions are less affected by the magnetic field. Thus, electron current is collected primarily along the magnetic field, and the electron sheath should be extended along the magnetic field lines. The spacecraft velocity $v$ is larger than the ion thermal velocity and smaller than the electron thermal velocity. For such conditions, formation of a wake behind the body is expected (Al'pert et al., 1965). We note, in passing, that the ion 

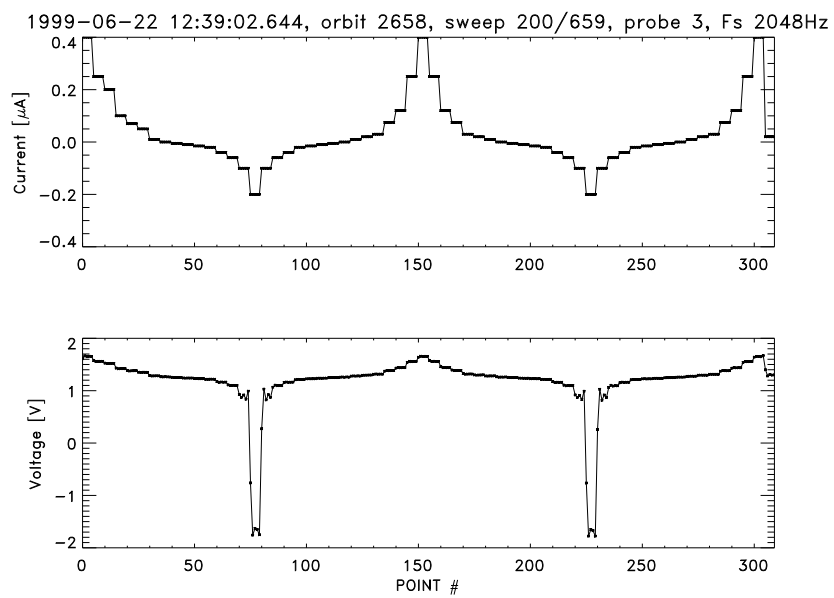

Fig. 2. An example of a multiple EMMA sweep. The upper panel presents the bias current versus sample number, going down and up between -0.2 and $0.4 \mu \mathrm{A}$ twice; the lower panel shows the measured probe potentials for the same samples. Sampling frequency was $2048 \mathrm{~Hz}, 5$ samples with each bias current value were taken.

current collected by the satellite will be determined by the projected area transverse to $\boldsymbol{v}$. Finally, the illumination by the Sun creates an asymmetry in the photoelectron emission.

The last aspect is probably the least important, as the Astrid-2 spin axis was kept within $30^{\circ}$ around the Sun's direction, in order to keep the solar panels illuminated. This means that the EMMA and LINDA probes in the spin plane were never shadowed by the satellite body. The $\boldsymbol{v}$ and $\boldsymbol{B}$ orientations change during the course of orbital motion. The orbital plane and the spin plane of the satellite normally were not parallel. Thus, $\boldsymbol{v}$ lies in the spin plane twice per orbit, mostly at low latitudes. In some cases, when the angle between the two planes is small (when the orbit goes in the dusk-dawn direction), $v$ may be close to the spin plane all the time. The ambient magnetic field $\boldsymbol{B}$ is almost identical to the Earth's intrinsic magnetic field, which is close to dipolar. Therefore, $\boldsymbol{B}$ is in the spin plane four times per orbit - twice at low latitudes and twice at high latitudes, in the opposite hemispheres.

We can expect to detect the effects of the complex sheath of the satellite on the available probes only for specific relative orientations of $\boldsymbol{v}$ and $\boldsymbol{B}$. At low latitudes, the satellite travels almost along the magnetic field lines and both the $\boldsymbol{v}$ and $\boldsymbol{B}$ directions are typically close to the plane where the probe measurements are done. At high latitudes, the angles between $\boldsymbol{v}$ and $\boldsymbol{B}$, and $\boldsymbol{v}$ and the spin plane are determined by the orbital plane orientation, the tilt of the Earth's magnetic dipole axis and direction of the satellite spin axis. We also expect the sheath size and structure to depend on the plasma parameters, such as density and temperature.

At any single time the measured quantities are only four EMMA probe potentials and two currents collected by the LINDA probes. However, by combining the measurements from a spin period, a one dimensional characteristic along the probe trajectory around the satellite (as a function of spin

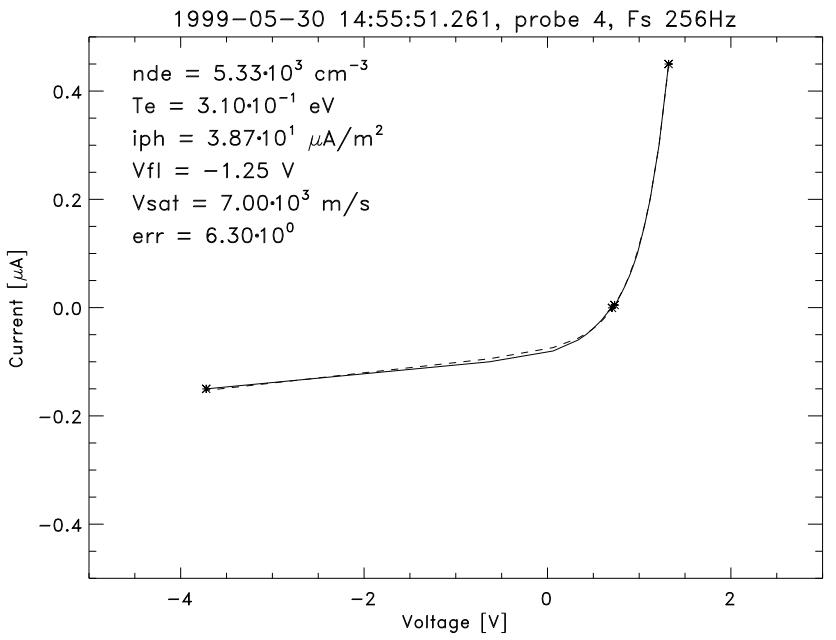

Fig. 3. An example of current sweep on an EMMA probe with a theoretical curve fitted to it. Solid curve - measured data, dashed curve - theoretical dependence (see text).

phase $\phi$ ) can be obtained, assuming the controlling parameters (plasma density and temperature) do not change much during the spin period. Using the changes of the orientation of $\boldsymbol{B}$ and $\boldsymbol{v}$ with respect to the spin plane, one may obtain a two dimensional cut through the sheath structure by a sphere with a radius equal to the probe distance from the satellite centre. This assumes the plasma parameters to be constant throughout the whole interval needed to cover the ranges of interest in both the spin phase, and the angle between the spin plane and the magnetic field.

\section{Multi-instrument measurements of disturbances of the plasma environment}

The EMMA instrument operates the plasma probes in the high impedance mode, i.e. the potentials $V_{i}$ of the four probes relative to the satellite are measured for a set bias current

$V_{i}=\phi_{i}-\phi_{s a t}$

where $\phi_{i}$ is the potential of the $i$-th probe, and $\phi_{s a t}$ is the satellite potential. The potential of each probe depends on its environment

$\phi_{i}=\phi_{p l}+\delta \phi_{i}\left(I_{\text {bias }}, n, T, \ldots\right)$

where $\phi_{p l}=-\boldsymbol{E} \cdot \boldsymbol{r}_{i}$ is the local plasma potential relative to the satellite location due to the presence of electric field $\boldsymbol{E}$, and $\delta \phi_{i}$ is a combination of the "contact" potential of the probe, which is a function of bias current $I_{\text {bias }}$, plasma parameters $(n, T, \ldots)$, and the disturbance of the plasma potential due to the satellite complex sheath. In the double probe method for measuring the electric field, it is required that the potentials of the probes are close to the local plasma potentials $\left(\delta \phi_{i} \approx 0\right)$, or that the potential differences between plasma and the probes are the same for all the probes $\left(\delta \phi_{i}=\delta \phi\right)$. To achieve this, the probes are made as identical 

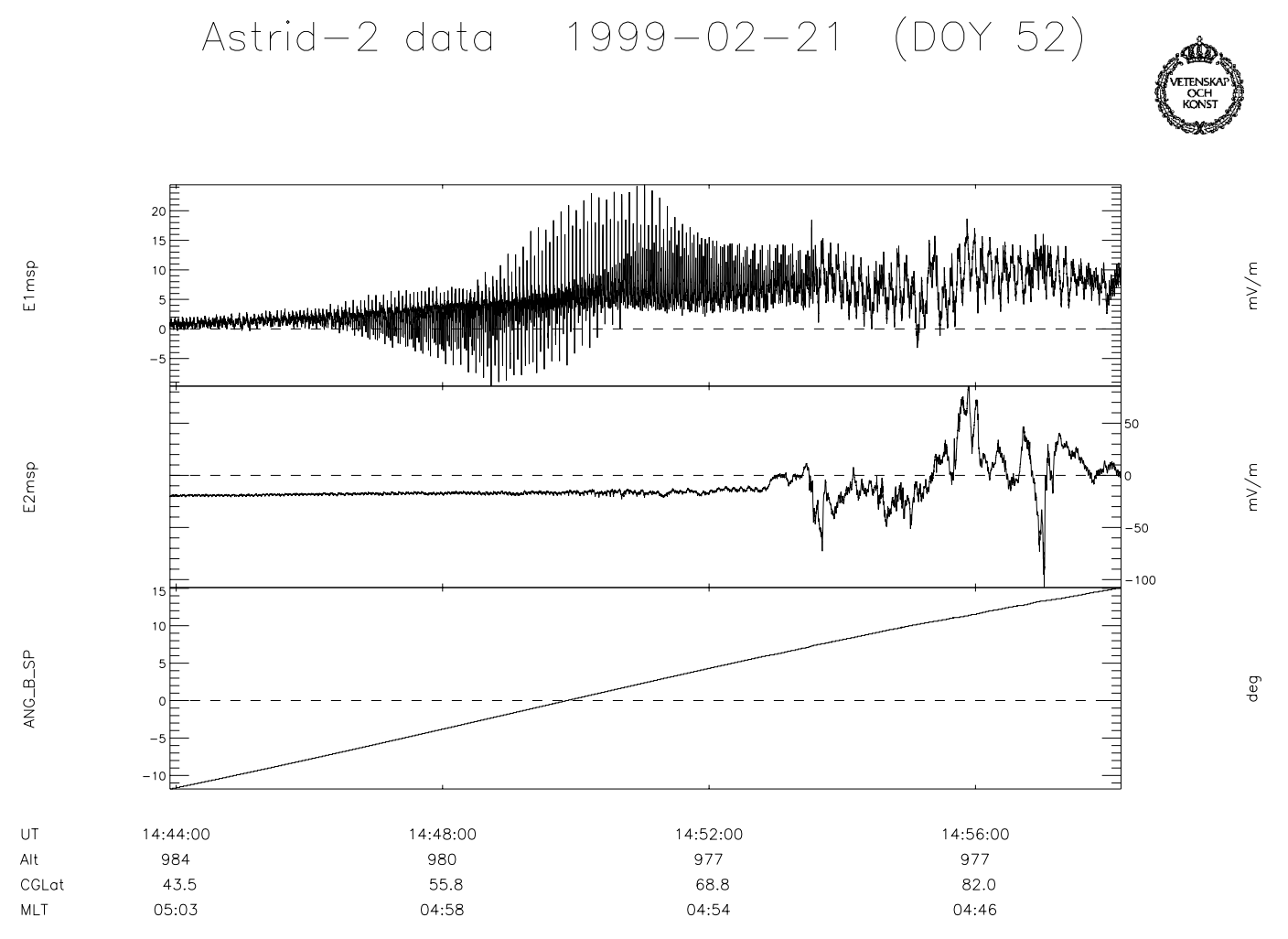

Fig. 4. Spurious electric fields observed when the magnetic field lies in the satellite spin plane. Panels from top to bottom show the components of the measured electric field in the spin plane most parallel and perpendicular to the ambient magnetic field, and the angle between the magnetic field and the spin plane.

as possible, and fed with a positive bias current to keep the probes close to the plasma potential. By taking the difference of the potentials of opposite probes, the potential drop in the plasma is estimated:

$\Delta V_{i j}=V_{i}-V_{j}=\phi_{i}-\phi_{j}$

and by dividing it by the distance between the probes, the electric field is obtained. Due to the motion of the satellite across the geomagnetic field lines, an induced electric field $\boldsymbol{E}=\boldsymbol{v} \times \boldsymbol{B}$ is set up in the satellite reference frame. This field can be as large as $350 \mathrm{mV} / \mathrm{m}$ for low altitude ionospheric satellites, such as Astrid-2 and thus, is often the main contribution to the measured electric field. In order to study the physically interesting electric fields, it is subtracted from the measurements.

The presence of the satellite may disturb both the potential distribution in the plasma and the properties of the plasma itself (density and temperature), which determine $\delta \phi_{i}\left(I_{\text {bias }}, n\right.$, $T, \ldots)$. The changes in plasma parameters may be different at the locations of different probes. Thus, subtracting the signals from the opposite probes will introduce a non-cancelled term of $\delta \phi_{i}-\delta \phi_{j}$ into (3), leading to a spurious contribution to the signal unrelated to the ambient electric field. A known example is the spurious parallel electric field as seen when the probes pass the magnetic field lines connected to the satellite (see Fig. 4). When the angle between $\boldsymbol{B}$ and the spin plane crosses zero, a "hedgehog" of spikes in the elec- tric field component parallel to $\boldsymbol{b}$ is observed. This effect will be discussed later in the paper.

To analyze the disturbances related to the complex sheath of the satellite, the differential probe signal is taken to get rid of variations of the spacecraft potential. The plasma environment is expected to be disturbed in limited ranges of angles around specific directions of the probe booms (e.g. spin phases when the booms are aligned with $\boldsymbol{B}$ or $\boldsymbol{v}$ ). Thus, it is more convenient to take the difference of the adjacent probe potentials, e.g. $V_{12}=V_{1}-V_{2}$, shifting the disturbances by $90^{\circ}$ in the spin phase. (Probes 1 and 3 are both in the disturbed regions for the same spin phase).

The $V_{12}$ signal is a sine with an amplitude determined by the contributions due to $\boldsymbol{v} \times \boldsymbol{B}$, and any geophysical electric field that may exist in the ionosphere. In addition, satelliterelated disturbances appear for the specific spin phase intervals. It is found that the strongest disturbances are seen when a probe is close to the magnetic field lines connected to the satellite body. To concentrate on the latter signal, we subtract a sine fit to the data, with a period equal to the spin period. Only spin phase intervals, where no probe is within $15^{\circ}$ from the magnetic field direction, are used in the fitting. The residual may be interpreted as $\delta \phi_{i}$.

The current to the LINDA probe depends on the plasma properties, primarily on electron density and temperature, 
and potential relative to the plasma

$I_{n 2}=I\left(\phi_{n 2}, n, T, \ldots\right)$.

While the probe potential is kept constant at $U_{\text {bias }}=+10 \mathrm{~V}$ relative to the satellite (in the reference frame moving with the satellite), it varies relative to the plasma

$\phi_{n 2}=\phi_{s a t}+\boldsymbol{r}_{n 2} \cdot(\boldsymbol{v} \times \boldsymbol{B})+U_{\text {bias }}$

where $\phi_{n 2}$ is the potential of the LINDA probe $n 2$ relative to the plasma at the location of the probe, $\phi_{\text {sat }}$ is the potential of the satellite relative to the plasma at the satellite's location, and $\boldsymbol{r}_{n 2}$ is the position of the probe relative to the satellite. The assumption normally made in order to relate the LINDA current $I_{n 2}$ to the plasma density is that both $\phi_{\text {sat }}$ and the $\boldsymbol{v} \times \boldsymbol{B}$ contribution are much smaller than $U_{\text {bias }}$, and $\phi_{n 2}$ does not change much with respect to the plasma. In many cases, this is valid, as will be shown for specific cases. The variations of $I_{n 2}$ are then related to the plasma parameters. For a Maxwellian plasma, the dependence on the density is linear, while the temperature dependence is proportional to the square root. Thus, it is a good approximation to assume most of the time that the temperature is constant, and the collected current is a measure of the electron density.

Disturbances of the EMMA probe potentials are difficult to unambiguously relate to the plasma parameter changes. Interpretation of the LINDA probe current as being proportional to the electron density is based on assumptions of isotropic plasma at constant temperature. The plasma in the complex sheath of a satellite may be anisotropic and nonMaxwellian. The most complete information to be obtained with the probe measurements is the current-voltage characteristics of the probe in plasma; but even then, one has to resort to models describing the current collection by the probe.

The EMMA electric field instrument was equipped with a sweep function, which allowed one to switch the bias current to a probe, according to a table uploaded from the ground. The maximum sweeping rate was 4 sweeps/second. However, by making the bias current go up and down several times in the sweep table, multiple physical sweeps within one logical sweep could be carried out (see Fig. 2), thus making it possible to determine the plasma current-voltage characteristics at multiple occasions during the spin period. While representing the most interesting mode for studying the satellite sheath, this mode required extra commanding of the satellite and used much of the on-board memory (it requires sampling the probe potentials at the 2048 samples/s, which fills the memory in 4.5 minutes), and disturbed the electric field measurements by taking one of the probes out of the four-probe configuration. This special mode has been commanded on several occasions in June and July 1999.

A sweep analysis tool was developed to automatically fit a theoretical current-voltage curve to the measured sweep (see Fig. 3). In the simplest model (e.g. Lindqvist et al., 1994) the current to a probe can be written as

$I_{\text {bias }}=4 \pi r^{2} n_{e} e \sqrt{\frac{k T_{e}}{2 \pi m_{e}}}\left(1+\frac{e V}{k T_{e}}\right)-\pi r^{2} i_{p h} e^{-e V / k T_{p h}}(6)$ for a positive probe and

$$
\begin{aligned}
& I_{\text {bias }}=4 \pi r^{2} n_{e} e \sqrt{\frac{k T_{e}}{2 \pi m_{e}}} e^{e V / k T_{e}}-\pi r^{2} i_{p h} \\
& -4 \pi r^{2} n_{e} e \sqrt{\frac{k T_{i}}{2 \pi m_{i}}+\frac{v_{s a t}^{2}}{16}}\left(1-\frac{e V}{8 m_{i}}\left(\frac{k T_{i}}{2 \pi m_{i}}+\frac{v_{s a t}^{2}}{16}\right)^{-1}\right)
\end{aligned}
$$

for a negative probe, where $r$ is the probe radius, $V$ is the potential of the probe relative to the plasma, $v_{s a t}$ is the velocity of the satellite, $i_{p h}$ is the photoemissivity, $k T_{p h}$ is the energy of photoelectrons, and the rest of the notations have their usual meaning. The equations are valid for an unmagnetised Maxwellian plasma streaming with a velocity $v_{\text {sat }}$ relative to the probe. As the potentials are measured relative to the satellite, a "floating" (satellite) potential should be introduced

$V_{i}=V+V_{f l}$.

The tool attempts to fit the theoretical curve by finding approximations for photoelectron current $i_{p h}$, electron density $n_{e}$, electron temperature $T_{e}$ and floating potential $V_{f l}$, and assuming nominal or typical values for the rest of the parameters. The complex relationship between the plasma parameters and the shape of the theoretical curve makes it difficult to use any standard fitting algorithms based on the least squares fit.

The algorithm used consists of the selection of four fixed points on the sweep curve, two on each of the two more or less straight parts, and an iterative loop which tries to fit the curve at these points. There are separate fitting functions for each of the four plasma parameters. One of them has an iterative loop in itself, others just take one iterative step each time the function is called from the main loop. At the end of the main loop, there is a call to a function that checks if the chosen fitting accuracy is achieved. If not, the loop continues until the counters reach maximum values and the algorithm gives up. After the end of the loop, a least squares error is calculated for all the datapoints. If the error value is small enough, the fitting is further improved in another function which calculates the least squares errors for all combinations of parameters plus/minus some predetermined delta. The combination resulting in the smallest error is chosen and this is repeated six times, bisecting deltas when needed.

\subsection{Case 1: Motion quasiparallel to the magnetic field}

Figure 5 presents the potentials of the EMMA probes, the potential difference between probes 2 and 4, the LINDA current to a Langmuir probe and the angle between the magnetic field and the spin plane for 3 minutes on 22 June 1999. The satellite was travelling at low latitude. Due to the high inclination of the orbit, the satellite moved essentially in the north-south direction, with the velocity being close to the direction of the magnetic field. In this case, the angle between $\boldsymbol{v}$ and $\boldsymbol{B}$ changed from $6^{\circ}$ to $22^{\circ}$ during the interval, and both these vectors were within $10^{\circ}$ of the spin plane. 


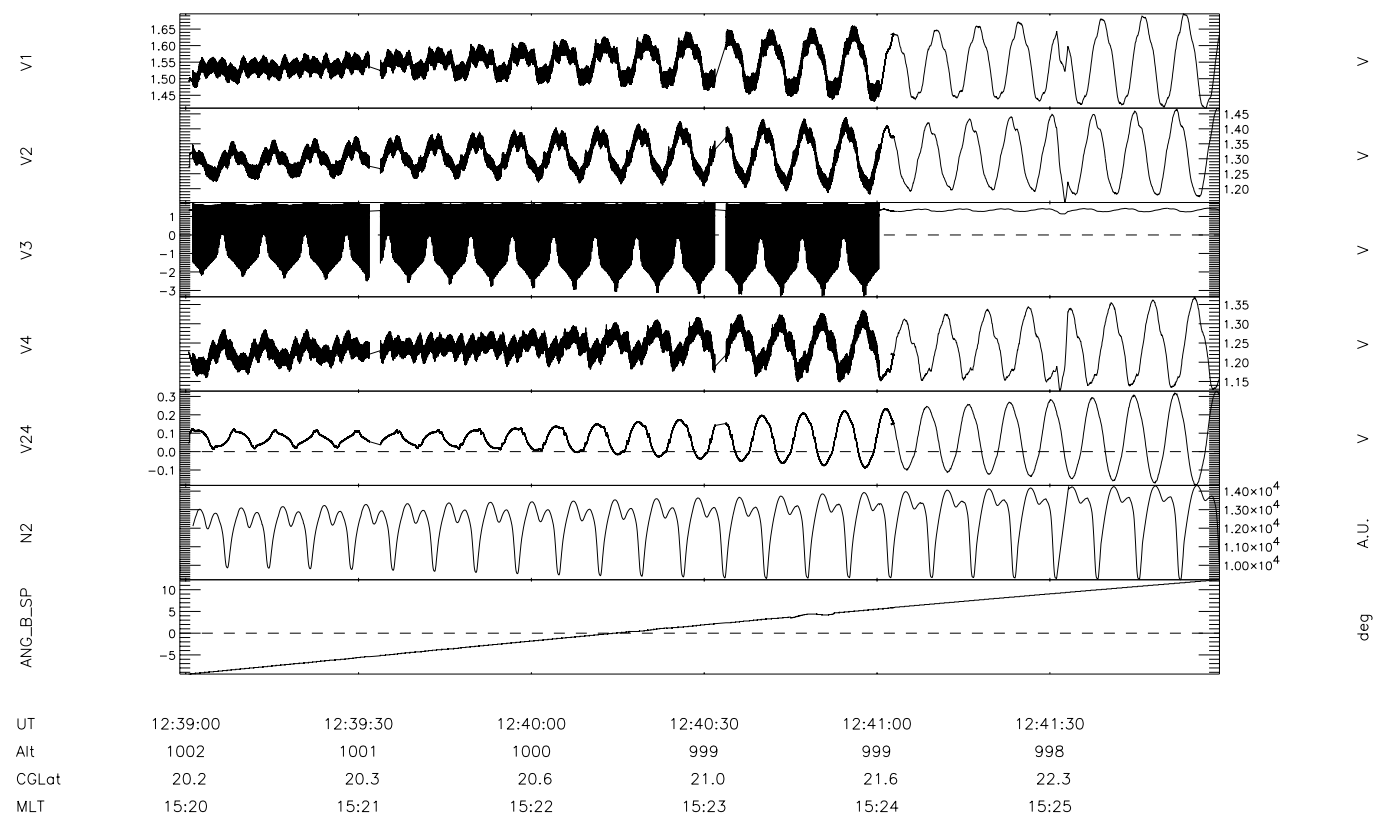

Fig. 5. EMMA measurements on 22 June 1999. Panels from top to bottom: potentials of probes 1 to 4 relative to the satellite, potential difference between probe 2 and probe 4, current collected by the LINDA probe, and angle $\alpha$ between $\boldsymbol{B}$ and the spin plane. Note the different scale in panel 3.

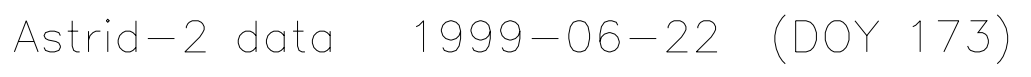

Orbit 7000
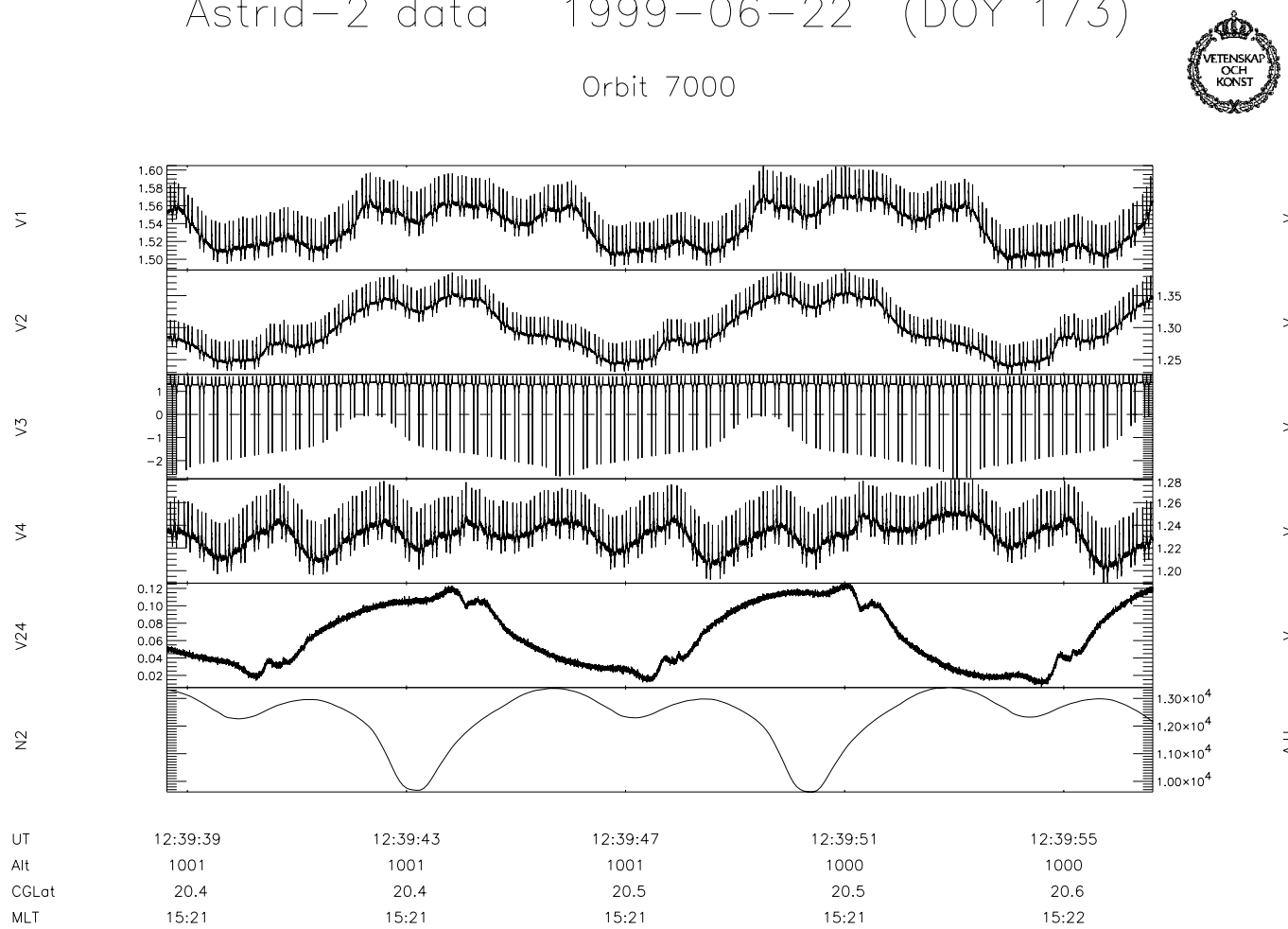

Fig. 6. Two spin periods of the interval in Fig. 5. The panels are the same as the top six panels of Fig. 5. 

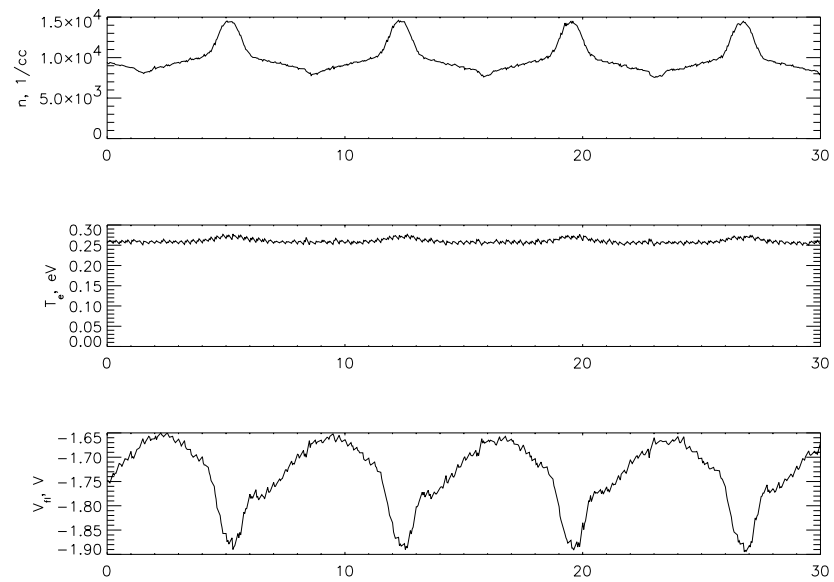

Fig. 7. Plasma parameters derived from the fits to the EMMA current sweeps for case 1. Panels from top to bottom: electron density, electron temperature and floating potential. Time interval of $30 \mathrm{sec}-$ onds spans four spin periods.

The EMMA instrument was operated in the sweep mode from 12:39 to 12:41 UT. Current sweeps on probe 3 are visible, as fast changes of the potential of that probe (see Fig. 6). It should be noted here, that the potential of the satellite itself is governed by a similar balance of currents as the potential of the probes. Changing the bias current to one of the probes requires modification of $\phi_{\text {sat }}$ to adjust the currents collected from the plasma in order to obtain a new balance. These changes of the satellite potential are seen in the $V_{1}, V_{2}$ and $V_{4}$ signals, although the potentials of these probes are not affected by sweeping probe 3 . The same effect is seen later at 12:41:30 UT, as a voltage sweep is carried out on the LINDA probes; all the $V_{i}$ signals show synchronous variations.

The differential signal $V_{24}=V_{2}-V_{4}$ is not affected by the sweeping and shows a quasi-sinusoidal oscillation of increasing amplitude, mainly caused by the $\boldsymbol{v} \times \boldsymbol{B}$ contribution. Due to the motion almost parallel to the magnetic field, this contribution is rather small. A closer inspection of the data on a smaller time scale reveals periodic irregularities in $V_{24}$. The LINDA probe current shows a spin phase dependence, as does the lower envelope of the probe 3 potential during the sweeps. Figure 7 presents plasma parameters obtained from the fits of the sweeps. The periodic variations of the estimated plasma density are evident, together with $V_{f l}$ variations and somewhat smaller temperature changes.

To understand the relation of the disturbances to the directions of $\boldsymbol{B}$ and $\boldsymbol{v}$, it is convenient to plot them in $(\phi, \alpha)$ coordinates, where $\phi$ is the spin phase measured from the position when probe 3 is aligned with the projection of $\boldsymbol{B}$ on the spin plane, and $\alpha$ is the angle between $\boldsymbol{B}$ and the spin plane. The direction of $\boldsymbol{v}$ changes somewhat during the interval, and can be described by two angles: an angle $\alpha_{v}$ between the spin plane and the $v$ direction, and $\phi_{v}$, the angle between the projections of $\boldsymbol{v}$ and $\boldsymbol{B}$ in the spin plane. Figure 8 presents the plasma parameters derived from the sweep fitting in $(\phi, \alpha)$ coordinates.

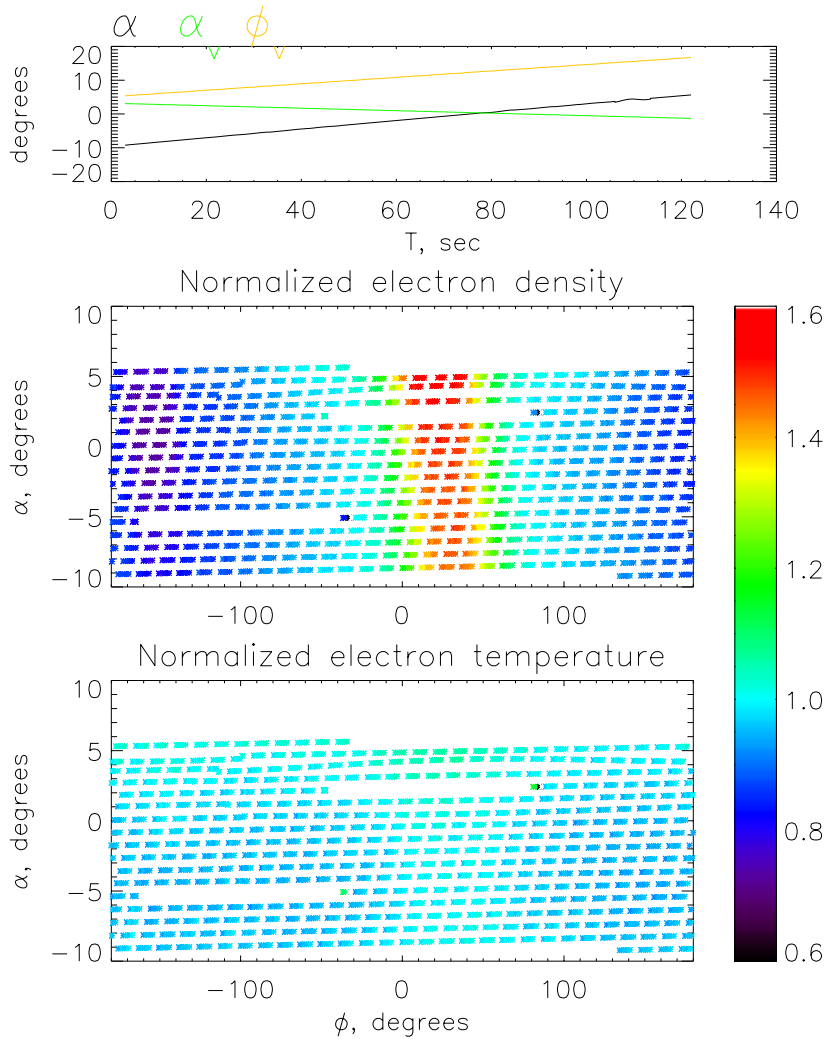

Fig. 8. "Maps" of plasma parameters in the vicinity of the Astrid-2 satellite, derived from the sweep measurements between 12:39 and 12:41 UT. The upper panel presents variation of the angle between $\boldsymbol{B}$ and the spin plane $\alpha$ during the period when the sweep data were taken, the angle between $v$ and the spin plane $\alpha_{v}$, and the angle between the projections of $\boldsymbol{B}$ and $\boldsymbol{v}$ in the spin plane $\phi_{v}$. Two panels below show normalized electron density and electron temperature as functions of spin phase $\phi$ and $\alpha$.

The estimated electron density lies between 8000 and $14000 \mathrm{~cm}^{-3}$, and the temperature around $0.25 \mathrm{eV}$. These are rather typical values for Astrid-2 alititudes. The density corresponds to the Debye length of 3 to $4 \mathrm{~cm}$, which is much less then the scale size of the satellite. The changes of the derived electron density are systematic and are almost a factor of two in the course of a spin period. Increase in the plasma density is observed for the angles when the swept probe is upstream of the satellite, and a narrower and more shallow density depletion is seen in the direction antiparallel to $\boldsymbol{v}$. A small temperature increase is coincident with the density increase, and probably indicates some heating in the ram. The floating potential shows a harmonic variation due to $\boldsymbol{v} \times \boldsymbol{B}$, a dip related to the density increase in the ram and some smaller irregularities at spin phases corresponding to the direction of the wake.

The sixth panel in Fig. 5 shows the current collected by probe 2 of the LINDA instrument, which is also plotted in $(\phi, \alpha)$ coordinates (here $\phi=0$ corresponds to probe $n 2$ boom being parallel to $\boldsymbol{B}$ ) in Fig. 9. In this case, the changes in $\phi_{\text {sat }}$ and $\boldsymbol{v} \times \boldsymbol{B}$ are both below $0.3 \mathrm{~V}$, so the basic assump- 

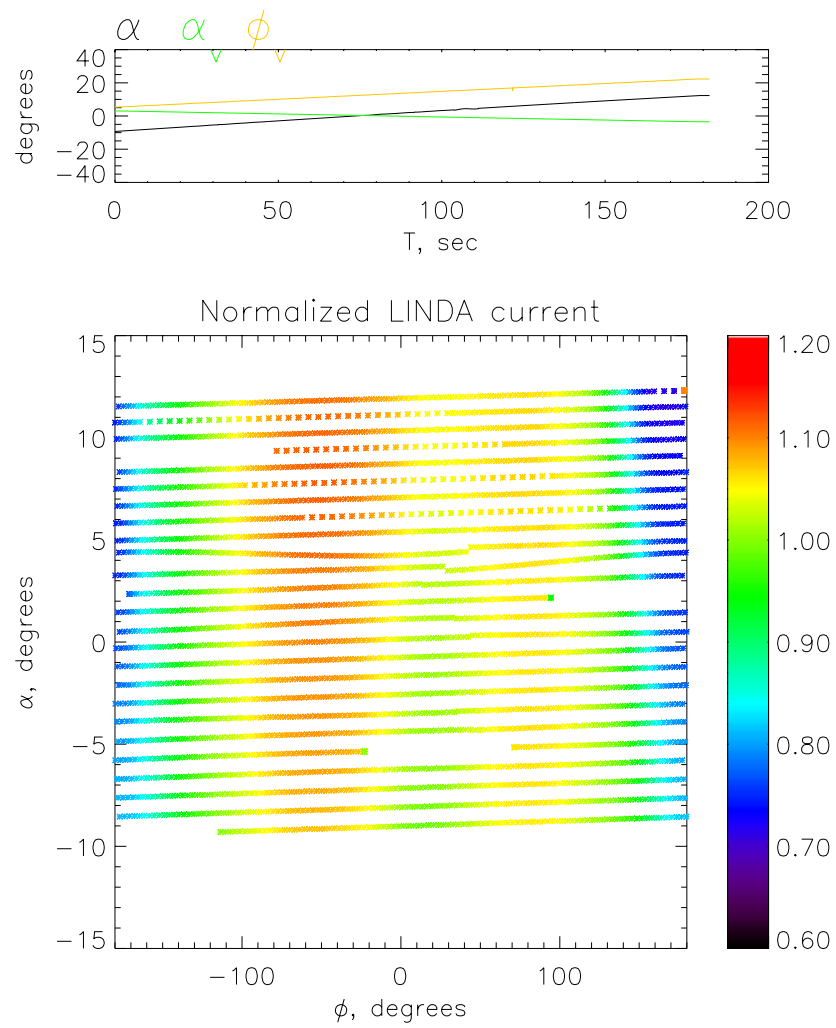

Fig. 9. Map of the current collected by LINDA probe $n 2$ (normalized) in $(\phi, \alpha)$ coordinates. In this figure $\phi=0^{\circ}$ corresponds to LINDA $n 2$ probe boom being parallel to $\boldsymbol{B}$.

tion of constant bias potential relative to the plasma is valid. No absolute calibration is used here to relate probe current to plasma density. In this figure the most prominent feature is the current decrease in the wake region around $\phi=-180^{\circ}$. The compression in the ram region is no longer symmetric as it was for the EMMA probe, but rather shows two broad maxima, with the leading one being somewhat higher (see also Fig. 5). Surprisingly enough, the direction of the ram proper corresponds rather to the local minimum. Another unexpected result is that the total range of the variation of the current to the probe is smaller than that of the density estimates from the sweeps on EMMA probes (located further from the satellite), while the ram compression is expected to be decreasing away from the body.

A $(\phi, \alpha)$ map of the distribution of the EMMA probe differential potential $V_{12}$ with a sine fit subtracted is presented in Fig. 10. Four regions of disturbed potential are clearly visible: at $0^{\circ}, 90^{\circ},-180^{\circ}$ and $-90^{\circ}$. The region at $0^{\circ}$ corresponds to the probe 1 boom being antiparallel to $\boldsymbol{B}$, and at $-180^{\circ}$ along the magnetic field. The probe 2 boom is parallel to the magnetic field at $90^{\circ}$. Note that a positive potential disturbance seen on probe 1 gives $V_{1}-V_{2}>0$, and on probe 2, gives $V_{1}-V_{2}<0$. The changes of the potential are mainly positive. The disturbance in the ram of the satellite ( $\boldsymbol{v}$ is approximately along $\boldsymbol{B}$ ) is broader, and less structured. The disturbance in the wake of the satellite is rather local-

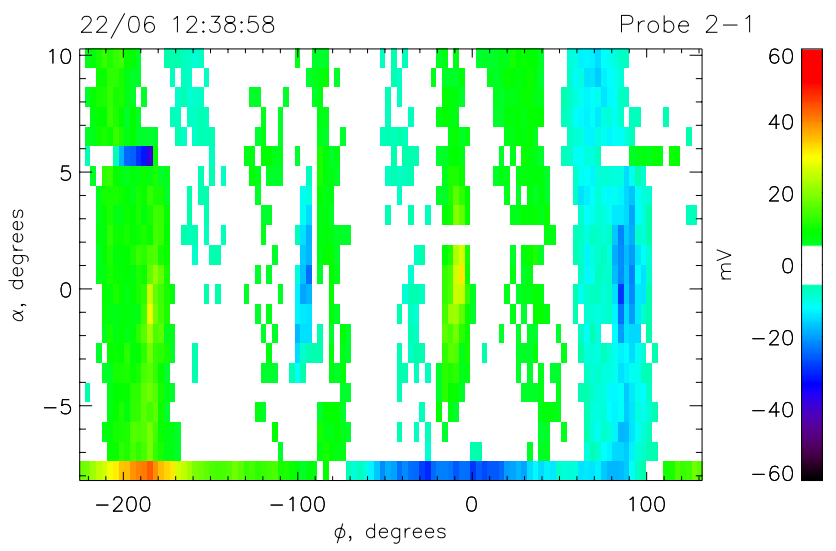

Fig. 10. Map of the disturbances of the potentials of EMMA probes 1 and 2 given as $V_{1}-V_{2}$ in $(\phi, \alpha)$ coordinates. Probe 1 is parallel to $\boldsymbol{B}$ at $\phi=-180^{\circ}$, and probe 2 is parallel to $\boldsymbol{B}$ at $\phi=90^{\circ}$.

ized to about $15^{\circ}-20^{\circ}$ around the direction of $-\boldsymbol{B}$ (a similar localized disturbance seems to be present even in the ram direction, masked by the broader maximum described). The amplitudes of the potential disturbances are of the order of several tens of $\mathrm{mV}$.

\subsection{Case 2: Motion oblique to the magnetic field}

Figure 11 presents the EMMA and LINDA data collected at 15:23:00 to 15:27:00 UT on 29 June 1999. As in case 1, the satellite was travelling at low latitudes and the velocity was close to the spin plane. The angle between $\boldsymbol{v}$ and $\boldsymbol{B}$ changed from $156^{\circ}$ to $136^{\circ}$ in the course of the interval, i.e. velocity was oblique to the magnetic field.

The results of the sweep fitting are presented in Fig. 12. The estimated density is about half the density for the interval considered in the first case. Figure 13 shows $(\phi, \alpha)$ the map of variations of plasma parameters derived from the sweep measurements. The total change in density during the spin is also about a factor of two, as in the first case. The shape of the changes is, however, somewhat different from the previous case. The density increase in the ram is broader and covers almost half of the spin period, while the wake region hardly shows any depression at all and is less symmetric with respect to the $\boldsymbol{B}$ direction, as compared to the first case. The temperature shows larger variations, also lacking a symmetry with respect to $\boldsymbol{B}$. The variations in $V_{f l}$ are dominated by the $\boldsymbol{v} \times \boldsymbol{B}$ variation, as the angle between $\boldsymbol{v}$ and $\boldsymbol{B}$ is larger in this case.

LINDA current is more difficult to analyze in this case, as the $\boldsymbol{v} \times \boldsymbol{B}$ contribution is larger than in the first case. We note, in passing, that peak-to-peak variation of the current during a spin period is on the order of $60 \%$, which is again less than the peak-to-peak change in the EMMA sweep derived density. 


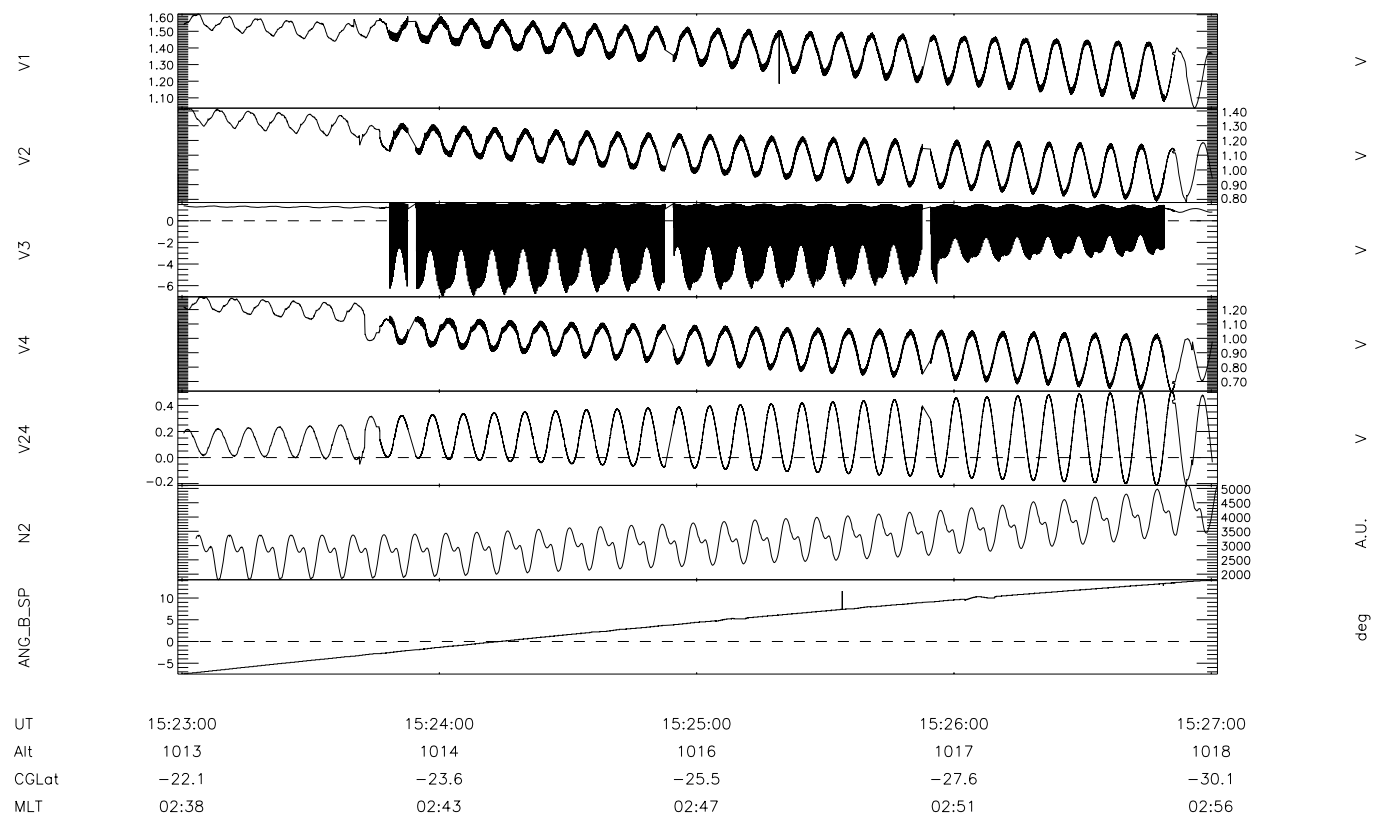

Fig. 11. EMMA measurements on 29 June 1999. The format is the same as in Fig. 5.
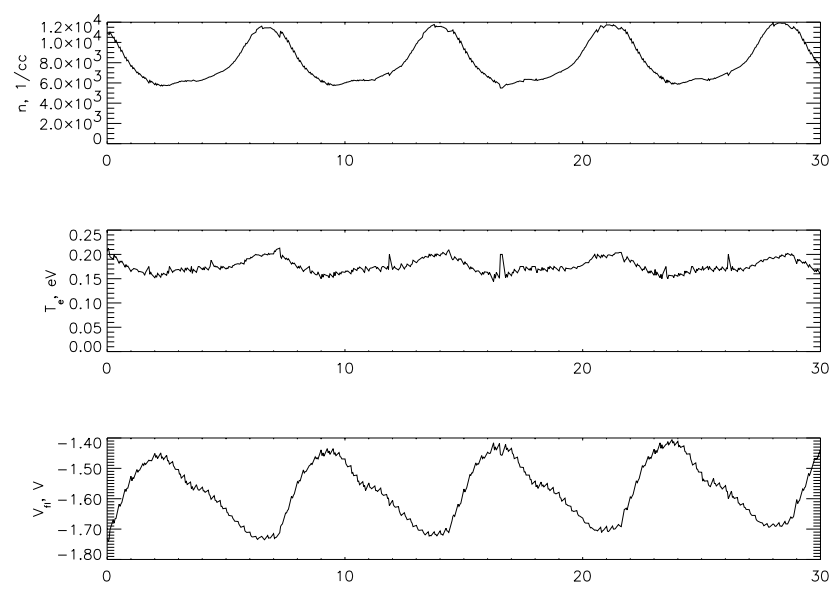

Fig. 12. Plasma parameters derived from the fits to the EMMA current sweeps for Case 2. The format is the same as in Fig. 7.

3.3 Case 3: Motion quasi-perpendicular to the magnetic field

The third case we present corresponds to the satellite moving at high latitudes. EMMA and LINDA data from 12:22:00 to 12:24:00 UT on 24 June 1999 are presented in Fig. 14. The angle between $\boldsymbol{v}$ and $\boldsymbol{B}$ changed from $92^{\circ}$ to $94^{\circ}$ in the interval, with the velocity making an angle of $40^{\circ}$ to the
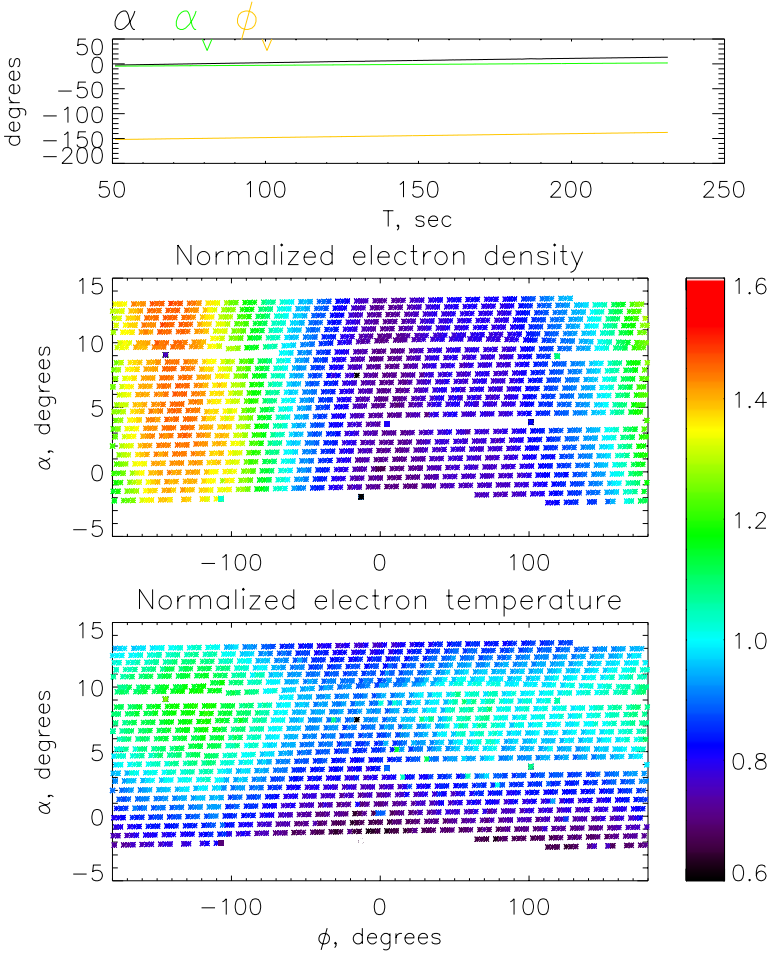

Fig. 13. Map of plasma parameters derived from the sweep measurements for Case 2 in $(\phi, \alpha)$ coordinates. The format is the same as in Fig. 8. 


$$
\text { Astrid-2 data 1999-06-24 (DOY 175) }
$$
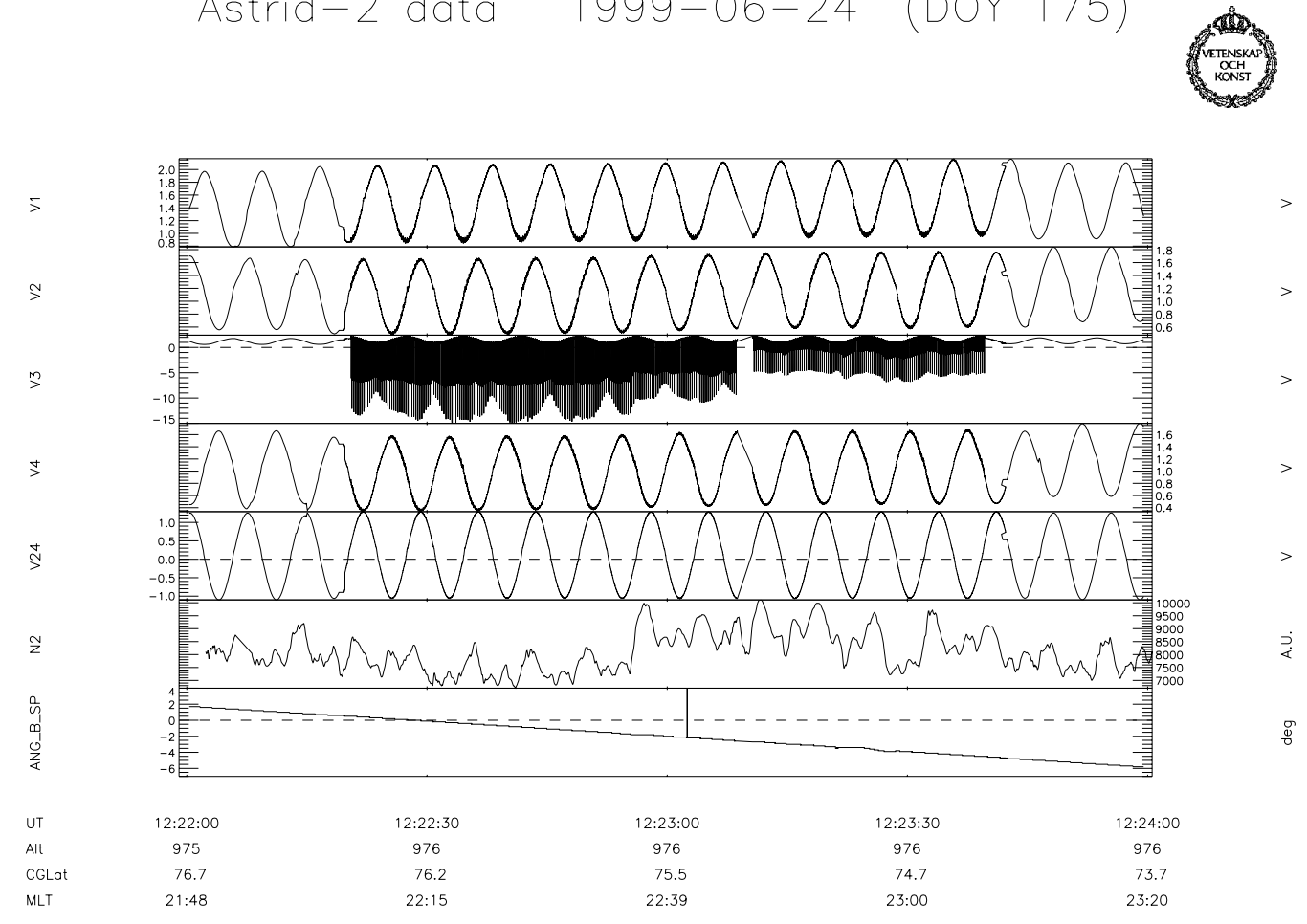

Fig. 14. EMMA measurements for Case 3. The format is the same as in Fig. 5.
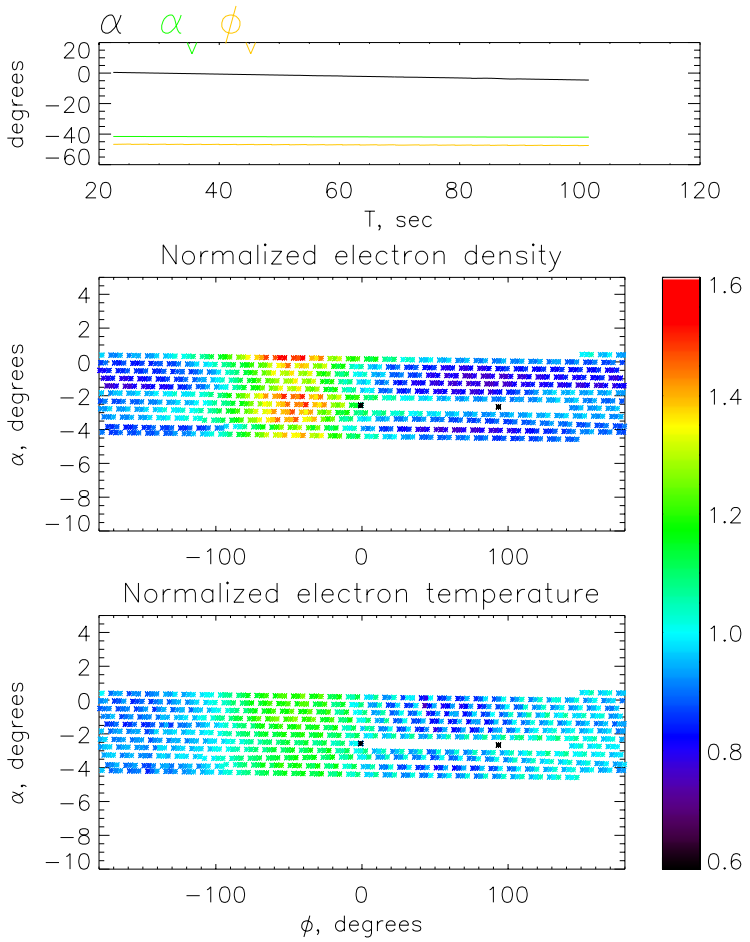

Fig. 15. Map of plasma parameters in $(\phi, \alpha)$ coordinates for Case 3. The format is the same as in Fig. 8. spin plane. The region is characterized by a rather disturbed density and larger $\boldsymbol{v} \times \boldsymbol{B}$ variations. This makes analysis of the LINDA current data less straightforward, but systematic variations with several local minima and maxima during the spin period are clearly seen in the figure.

Figure 15 presents a $(\phi, \alpha)$ map of the sweep-derived plasma parameters. A compression in the ram region is seen again, although the velocity vector is quite far away from the spin plane.

\subsection{Cases 4 and 5: Disturbances in the probe potentials}

In the following two cases, no sweep measurements were made by the EMMA instrument. On the other hand, the disturbances of the electric field probe potentials are very typical here. A large number of cases, where only continuous EMMA measurements in normal mode were available, was analyzed by Facciolo (2000).

Figure 16 presents the $(\phi, \alpha)$ map of the disturbance in the differential potential $V_{12}$ observed at around 00:56 UT on 14 March 1999. The satellite was travelling at low latitudes, i.e. the case is similar to case 1. The $\boldsymbol{v}$ and $\boldsymbol{B}$ directions are close throughout the interval. A triangle-shaped positive disturbance of $V_{12}$ is clearly visible around $0^{\circ}$ and $-90^{\circ}$ (when probes 1 and 2, respectively, are in the wake), while another much broader unstructured maximum is seen around $90^{\circ}$ and 


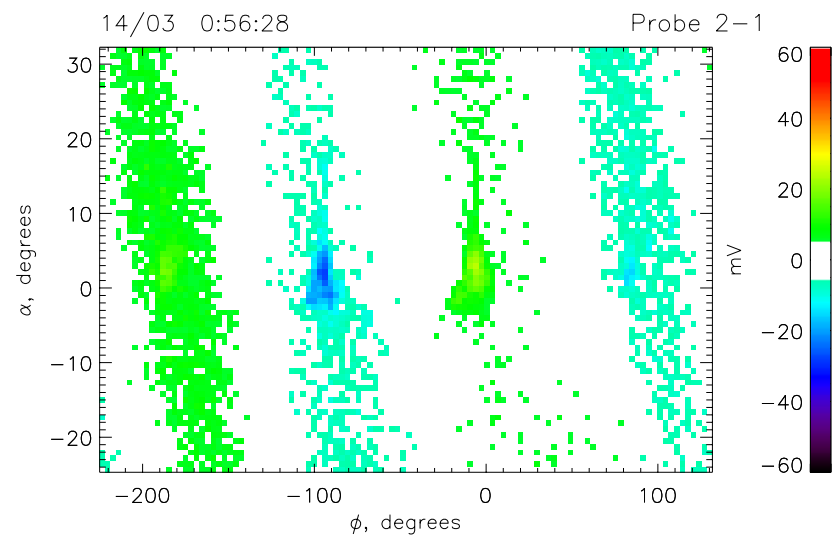

Fig. 16. $(\phi, \alpha)$ map of disturbances in the EMMA probes potentials fo Case 4 . Broad smooth increases are evident at $-180^{\circ}$ when probe 1 is ahead of the satellite, and at $90^{\circ}$, when probe 2 is ahead of the satellite. In the regions behind the satellite, narrower and more structured increases are seen at $0^{\circ}$ and $-90^{\circ}$, respectively.

$-180^{\circ}$ (in the ram region). The shape of the former disturbance is similar to the satellite shape seen in perspective from the location of the probes; the part narrow in $\phi$ extends long in the positive $\alpha$ direction, corresponding to the stiff boom, and the part broader in $\phi$, is localized within several degrees of zero in $\alpha$, corresponding to the satellite body and solar panel (compare to Fig. 1).

Figure 17 presents the $(\phi, \alpha)$ map of the disturbance at around 12:10 UT on 8 March 1999. The satellite was travelling at high latitudes, with the velocity being almost perpendicular to the spin plane. The potential $V_{12}$ here is quite disturbed due to the presence of auroral electric fields, which is visible as noise in the map. The triangle-shaped disturbance of the potential is recognized again, although this time, it is observed on both occasions when a probe is close to the magnetic field line crossing the satellite. The signatures at $0^{\circ}$ and $-180^{\circ}$ are almost mirror images of each other, as are disurbances at $90^{\circ}$ and $-90^{\circ}$. As the velocity vector is far from the spin plane, we do not expect to see any signatures of wake or ram, so the disturbance must be solely related to the sheath or presheath of the satellite, extending along the magnetic field lines.

\section{Discussion and summary}

A study of disturbances of the plasma environment by the Astrid-2 microsatellite is presented. The presence of the satellite modifies plasma parameters. These disturbances can be detected by the onboard probes, and thus, lower the quality of the measurements if not treated properly. The complicated geometry of the system makes it difficult to correct for the sheath and wake effects.

The motion of the satellite is supersonic relative to the ion thermal velocity. Plasma is compressed in front of the satellite and a wake (rarefaction) forms behind it. The change in plasma density depends on the distance from the satellite

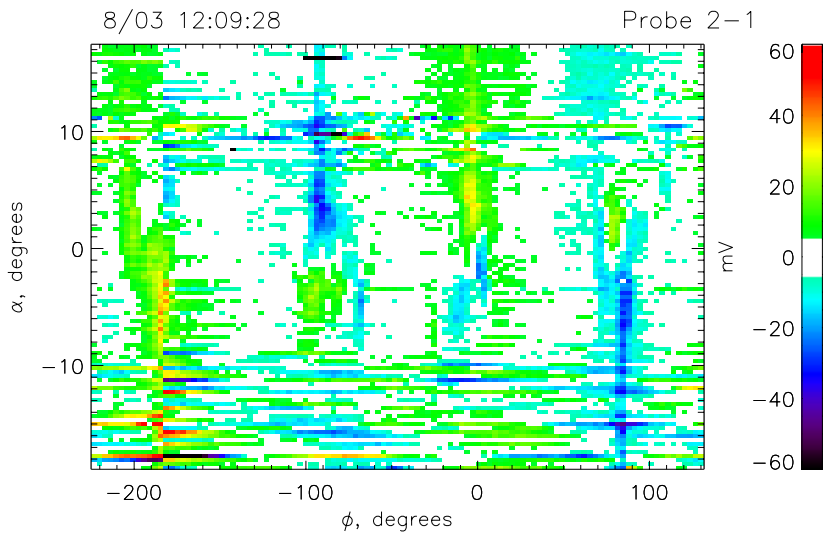

Fig. 17. $(\phi, \alpha)$ map of disturbances in the EMMA probes potentials for Case 5. Potential disturbances related to the electric field changes on timescales below the spin period are seen as noise throughout the figure. Structured disturbances in probe potentials are evident when the probes are close to the magnetic field direction.

body. An estimate of plasma density, derived from analyzing current sweeps from the EMMA probes located at $3.3 \mathrm{~m}$ from the satellite center, shows a compression of several tens of percent extending over tens of degrees around the satellite velocity direction, and a smaller density depression sometimes observed in the wake. The density derived from the LINDA probe (1.4 m from the satellite center) current measurements shows a different picture. A compression is also observed, but it is more structured, having broad local maxima not necessarily in the ram direction. A rather narrow wake region is found behind the spacecraft. However, the total range of variation of the electron density estimated at the EMMA probe locations (a factor of 2) is larger than the range of LINDA current variation (about $60 \%$ ). The compression in the ram direction dominates the EMMA sweep measurements, while the depletion the wake is clearly visible in the LINDA data.

These results are in consistence with theories by Al'pert et al. (1965), predicting density enhancements by tens of percent on distances of the order of the body scale size. The density enhancements are due to the reflection of the incoming ions from the satellite body. Various descriptions of the reflection process lead to different estimates of the density enhancements. The enhancement near the body is sensitive to the shape of the body and surface properties, whereas at larger distances, the details of the geometry are smeared out. On the other hand, stronger compression is expected close to the body, contrary to the fact that LINDA current shows smaller peak-to-peak density variation than EMMA sweeps.

Apart from the density modifications around the satellite, the plasma potential is modified in regions connected to the satellite by magnetic field lines. The changes in potential are of the order of tens of $\mathrm{mV}$ at the distance of the EMMA probes (which by far exceeds the Debye length for typical plasma conditions encountered by Astrid-2). The shape of the disturbance seems to follow the shape of the satellite, as 
seen from the probes. We attribute these potential disturbances to the presheath region of the satellite.

Due to the density modification by the satellite motion, the measurements of plasma density with the positively biased LINDA probes are subject to uncertainties of up to several tens of percent on time scales below the spin period. The presheath region, on the other hand, seems to have only a minor effect, if any, on this kind of measurement, as the changes of plasma potential are quite small compared to the bias potential. The picture is, thus, rather different than that of density measurements on the Viking satellite with biased Langmuir probes in low density space plasmas at higher altitudes (Hilgers and Holback, 1993). There, the spin modulation of current to a biased probe was caused by various regimes for collecting both the photoelectrons from the satellite, which were escaping along the magnetic field, and the plasma electrons. For small background densities, the dynamics of the photoelectrons from the satellite become very important, simultaneously as the Debye length becomes comparable to the scale size of the satellites. A common point is that the magnetic field seems to be important even for the Astrid-2 satellite.

In the electric field measurements, the effects of the presheath are more obvious. When a probe enters plasma regions connected by magnetic field lines to the satellite, its potential is changed by tens of $\mathrm{mV}$. As the satellite shape is not symmetric with respect to the magnetic field, this leads to the detection of spurious electric fields parallel to $\boldsymbol{B}$ with amplitudes of up to tens of $\mathrm{mV} / \mathrm{m}$. An example of this is presented in Fig. 4, and another possible example from the Freja satellite on similar altitudes was reported in Fig. 4 of Chust et al. (1998). This points out the intrinsic difficulties in measuring the parallel electric field by the double probe method. The parallel electric field measurements can be further affected by shadowing of directed particle fluxes by the satellite body, e.g. for measurements in auroral arcs. This question was not addressed in this study and only cases without significant particle precipitation were studied. In the case of extremely low densities or satellite charging, the sheath of the satellite may extend beyond the probes, producing even larger potential disturbances than the ones reported in this paper.

Acknowledgement. Topical Editor G. Chanteur thanks two referees for their help in evaluating this paper.

\section{References}

Al'pert, Yu. L., Gurevich, A. V., and Pitaevskii, L. P., Space Physics with Artificial satellites, Consultants bureau, New York, 1965.

Chust, T., Louarn, P., Volwerk, M., de Feraudy, H., and Roux, A., Electric fields with a large parallel component observed by the Freja spacecraft: Artifacts or real signals? J. Geophys. Res., 103, 215-224, 1998.
Dobrowolny, M. and Melchioni, E., Electrodynamic Aspects of the first tethered satellite mission, J. Geophys. Res., 98, 1376113778, 1993.

Eriksson, A. I. and Boström, R., Measurements of plasma density fluctuations and electric wave fields using spherical electrostatic probes, IRF scientific report 220, Uppsala, Sweden, 1995.

Facciolo, L., The interaction between electric field probes, spacecraft and plasma in the Earth's ionosphere, Royal Institute of Technology, Division of Plasma Physics Internal Report, ALP2000-103, 2000.

Fahleson, U., Theory of electric field measurements conducted in the magnetosphere with electric probes, Space Sci. Rev., 7, 238 262, 1967.

Fahleson, U. V., Fälthammar, C.-G., and Pedersen, A., Ionospheric temperature and density measurements by means of spherical double probes, Planet. Space Sci., 22, 41-66, 1974.

Garrett, H. B., The charging of spacecraft surfaces, in Handbook of geophysics and the space environment, ed. A. S. Jursa, US Air Force, 1985.

Hastings, D. E., A review of plasma interactions with spacecraft in low Earth orbit, J. Geophys. Res., 100, 14457-14483, 1995.

Hilgers, A., Holback, B., Holmgren, G., and Boström, R., Probe measurements of low plasma densities with applications to the auroral acceleration region and auroral kilometric radiation sources, J. Geophys. Res., 97, 8631-8641, 1992.

Hilgers, A. and Holback, B., Some aspects of satellite spin effects on sperical probe measurements in a magnetized plasma, Geophys. Res. Lett., 20, 347-350, 1993.

Hilgers, A., Interaction between biased sunlit electron collectors in an infinite-Debye-length magnetoplasma: Electron temperature threshold effect, J. Geophys. Res., 100, 5705-5713, 1995.

Holback, B., Jacksén, Å., Åhlén, L., Jansson, S.-E., Eriksson, A. I., Wahlund, J.-E., Carozzi, T., and Bergamn, J., LINDA - the Astrid-2 Langmuir Probe Instrument. Ann. Geophysicae, 19, 601-610, 2001 (this issue).

Kasha, M. A., The ionosphere and its interaction with satellites, Gordon and Breach, New York, 1969.

Laakso, H., Aggson, T. L., and Pfaff Jr, R. F., Plasma gradient effects on double-probe measurements in the magnetosphere, Ann. Geophysicae, 13, 130, 1995.

Lindqvist, P.-A., Marklund, G. T., and Blomberg, L. G., Plasma characteristics determined by the Freja electric field instrument, Space Sci. Rev., 70, 593-602, 1994.

Murphy, G. B. et al., The plasma wake of the Shuttle orbiter, J. Geophys. Res., 94, 6866-6872, 1989.

Neubert, T. et al., The sheath structure around a negatively charged rocket payload, J. Geophys. Res., 95, 6155-6165, 1990.

Samir, U. and Jew, H., Comparison of theory with experiment for electron density distribution in the near wake of an ionospheric satellite, J. Geophys. Res., 77, 6819-6827, 1972.

Samir, U. and Fontheim, E. G., Comparison of theory and in situ observations for electron and ion distributions in the near wake of the Explorer 31 and AE-C satellites. Planet. Space Sci., 29, 975-987, 1981.

Samir, U., Comfort, R. H., Singh, N., Hwang, K. S., and Stone, N. H., Insight into theory-experiment comparisons of wake measurements in the plasmasphere, Planet. Space Sci., 37, 873-880, 1989. 